\title{
Equivalence classes in the Weyl groups of type $B_{n}$
}

\author{
Thomas Pietraho
}

Received: 11 October 2006 / Accepted: 11 June 2007 / Published online: 6 July 2007

(C) Springer Science+Business Media, LLC 2007

\begin{abstract}
We consider two families of equivalence classes in the Weyl groups of type $B_{n}$ which are suggested by the study of left cells in unequal parameter Iwahori-Hecke algebras. Both families are indexed by a non-negative integer $r$. It has been shown that the first family coincides with left cells corresponding to the equal parameter IwahoriHecke algebra when $r=0$; the equivalence classes in the second family agree with left cells corresponding to a special class of choices of unequal parameters when $r$ is sufficiently large. Our main result shows that the two families of equivalence classes coincide, suggesting the structure of left cells for remaining choices of the IwahoriHecke algebra parameters.
\end{abstract}

Keywords Unequal parameter Iwahori-Hecke algebra · Domino tableaux · Robinson-Schensted algorithm

Mathematics Subject Classification (2000) 20C08 · 05E10

\section{Introduction}

Consider a Weyl group $W$ of type $B_{n}$, that is, the hyperoctahedral group $\mathbb{H}_{n}$. We will need two generalizations of the Robinson-Schensted algorithm. Let $S D T_{r}(n)$ be the set of standard domino tableaux of size $n$ and rank $r$. Then for every $r$, there is a map

$$
G_{r}: W \rightarrow S D T_{r}(n) \times S D T_{r}(n)
$$

which is a bijection of $W$ with its image, the set of same-shape pairs of standard domino tableaux (see [4] and [17]). Subsequently, we will write $\left(S_{r}(w), T_{r}(w)\right)$ for

T. Pietraho (凶)

Department of Mathematics, Bowdoin College, Brunswick, ME 04011, USA

e-mail: tpietrah@bowdoin.edu 
the pair $G_{r}(w)$. If we write $S B T(n)$ for the set of standard bitableaux of size $n$, then it is possible to define another algorithm

$$
H: W \rightarrow S B T(n) \times S B T(n) .
$$

The image of $H$ is the set of same shape pairs of standard bitableaux, and with this restriction, the map is again a bijection [16]. When $r \geq n-1$, there is a natural identification between the sets $S D T_{r}(n)$ and $S B T(n)$, and the corresponding bijections coincide.

As in [9], a Coxeter group $W$ and a weight function $L$ on $W$ can be used to partition $W$ into left cells. When $W$ is of type $B_{n}$, the weight function can be identified with a positive scalar $s$, and the description of the corresponding left cells is known when $s=1$, i.e. the equal parameter case [6], when $s=\frac{1}{2}$ and $s=\frac{3}{2}$ [2] and [8], and when $s>n-1$ [1]. We examine two families of equivalence classes on $W$ suggested by the above results in an effort to interpolate them for intermediate integer values of $s$.

We define the first equivalence relation on $W$ by letting $w \sim_{r} y$ iff the right tableaux in the image of $G_{r}, T_{r}(w)$ and $T_{r}(y)$, are related by moving through a set of open cycles. The second equivalence relation is defined using the right tableaux obtained from both $G_{r}$ and $G_{r+1}$. Let $w \varkappa_{r}^{\prime} y$ iff either $T_{r}(w)=T_{r}(y)$ or $T_{r+1}(w)=T_{r+1}(y)$ and take the equivalence relation $\varkappa_{r}$ to be its transitive closure. It is easy to see that for sufficiently large $r$, the two relations are the same. When $r \geq n-1$, the set of open cycles defining $\sim_{r}$ is necessarily empty, implying $w \sim_{r} y$ iff $T_{r}(w)=T_{r}(y)$, and further, that $w \sim_{r} y$ iff $w \varkappa_{r} y$. But even more is true.

Theorem 3.6 Fix a non-negative integer $r$. For elements $w, y \in W$, we have $w \sim_{r} y$ iff $w \leadsto r y$.

Both families of equivalence classes are derived from results on left cells of unequal parameter Iwahori-Hecke algebras for certain values of the parameter $s$. The relation $\sim_{0}$, as well as the notion of open cycles, was introduced by D. Garfinkle [4] and used to describe the left cells in the equal parameter case, i.e. when the scalar $s=1$. For all values of $r \geq n-1$, the equivalence classes of $\leadsto r$ in $W$ are the same, and correspond to the left cells in the unequal parameter case when $s>n-1$ (see [1]). This result of Bonnafé and Iancu originally described the left cells using right bitableaux of $H$, but it is easy to reconcile with this formulation by first, recalling the relationship between $H$ and $G_{r}$ for large $r$ and second, noting that $T_{r}(w)=T_{r}(y)$ iff $T_{r+1}(w)=T_{r+1}(y)$ for these values of $r$.

Since $\sim_{0}$ captures the left cell structure of $W$ when $s=1$ and $\leadsto r$ describes the left cells when $r=s-1>n-1$, the above theorem can be thought of as interpolating these results and suggests that $\sim_{r}$ and $\varkappa_{r}$ can be used to describe the left cell structure for intermediate integral values of $s$ as well. There is one more piece of evidence in support of this.

Bonnafé, Geck, Iancu, and Lam have conjectured that when $s$ is non-integral, the left cells are determined by the right domino tableaux for appropriate values of $r$ [2]. Lusztig's conjecture [9, (22.29)] as well as the propositions [9, (22.24)] and [9, (22.25)] then suggest that the common refinement $\rightarrow_{s-1}$ of these cells describes the 
left cell structure for integral values of $s$. The main result of this paper shows that this statement can be rephrased using the somewhat simpler equivalence relations $\sim_{s-1}$, and reconciles the conjecture with the original result of Garfinkle, Barbasch and Vogan when $s=1$.

Some comments concerning the geometrical considerations related to cells in this context are appropriate. First of all, according to Kazhdan-Lusztig theory, one would like to attach to each left cell a distinguished Weyl group representation together with a finite group. In the equal parameter case, this Weyl group representation is special in the sense of Lusztig and the finite group is the component group of the centralizer of an associated nilpotent orbit. In the asymptotic case, all cells are irreducible and one can take the corresponding finite groups to be trivial.

For the conjectured left cells in the intermediate cases, a choice of a distinguished Weyl group representation, or in other words, a choice of a distinguished partition among the shapes of tableaux of elements in the conjectured two-sided cell (see [2, Conjecture D]), is not clear. In the equal parameter case, the special representation attached to a left cell $\mathcal{C}$ corresponds to a partition which appears as a tableaux shape in all of the left cells contained in the two-sided cell of $\mathcal{C}$. Unfortunately, such a partition need not exist in the unequal parameter case. For instance, when $r=2$, no single partition appears among the tableaux shapes in every conjectural left cell contained in the conjectural two-sided cell defined from the partition $\left(4,3^{2}, 1\right)$.

It is possible, however, to attach a finite group to each conjectured left cell $\mathcal{C}$ in a manner reminiscent of the equal parameter case. The right tableaux of the involutions $\mathcal{C} \cap \mathcal{C}^{-1}$ are all related by moving through a subset of non-core open cycles. Hence $\mathcal{C} \cap \mathcal{C}^{-1}$ can be endowed with the structure of an elementary abelian group of order $2^{c}$, where $c$ is the number of non-core open cycles in the right tableaux of elements of $\mathcal{C}$. In the equal parameter case, McGovern has described the relationship of $\mathcal{C} \cap \mathcal{C}^{-1}$ to a quotient of the component group mentioned above [10].

Secondly, the map $G_{0}$, which classifies cells in the equal parameter case, is related to a more geometric Robinson-Schensted-type algorithm described in [11] and [14] which classifies orbital varieties in nilpotent orbits. The relationship between them is studied in [12], establishing a bijection between cells and the orbital varieties contained in special nilpotent orbits that is well-behaved with respect to a certain partial order. It would be interesting to investigate whether a link exists between $G_{r}$ for $r \neq 0$ and this geometric algorithm; however, because partitions of arbitrary rank do not naturally correspond to nilpotent orbits, the existence of such a relationship is not obvious.

Finally, Gordon and Martino have recently linked the combinatorics of cells in the unequal parameter case with the geometry of the Calogero-Moser space in [7]. There, the nilpotent points of Calogero-Moser space are shown to correspond to the combinatorially-defined conjectural two-sided cells.

\section{Preliminaries}

\subsection{Domino tableaux}

A Young diagram $D$ is a finite left-justified array of squares arranged with nonincreasing row lengths. A square in row $i$ and column $j$ of the diagram will be 
denoted $s_{i j}$ so that $s_{11}$ is the uppermost left square in the Young diagram below:



By $\partial(D)$ of $D$, we will denote the set of squares $s_{i j}$ of $D$ such that either $s_{i, j+1}$ or $s_{i+1, j}$ does not lie in $D$. We will also write $\rho(D)$ for the set of squares $s_{i, j+1}$ and $s_{i+1, j}$ that do not lie in $D$ but $s_{i j} \in \partial(D)$.

Let $r \in \mathbb{N}$ and $\lambda$ be a partition of a positive integer $m$; also write $\mathbb{N}_{n}=\{1,2, \ldots, n\}$. A standard domino tableau of rank $r$ and shape $\lambda$ is a Young diagram of shape $\lambda$ whose squares are labeled by elements of $\mathbb{N}_{n} \cup\{0\}$ in such a way that the integer 0 labels the square $s_{i j}$ iff $i+j<r+2$, each element of $\mathbb{N}_{n}$ labels exactly two adjacent squares, and all labels increase weakly along both rows and columns. We will write $S D T_{r}(\lambda)$ for the family of all domino tableaux of rank $r$ and shape $\lambda$ and $S D T_{r}(n)$ for the family of all domino tableaux of rank $r$ which contain exactly $n$ dominos. The set of squares labeled by 0 will be called the core of the tableau. We will write $\delta(T)$ for the set of $s_{i j}$ which satisfy $i+j=r+2$, and extend the notions of $\partial(D)$ and $\rho(D)$ to tableaux, writing $\partial(T)$ and $\rho(T)$.

\subsection{Generalized Robinson-Schensted algorithms}

We will consider the elements of the hyperoctahedral group $H_{n}$ as subsets $w$ of $\mathbb{N}_{n} \times \mathbb{N}_{n} \times\{ \pm 1\}$ with the property that the projections onto the first and second components of $w$ are always bijections onto $\mathbb{N}_{n}$. The element $w$ will be written as $\left\{\left(w_{1}, 1, \epsilon_{1}\right), \ldots,\left(w_{n}, n, \epsilon_{n}\right)\right\}$ and corresponds to the signed permutation $\left(\epsilon_{1} w_{1}, \epsilon_{2} w_{2}, \ldots, \epsilon_{n} w_{n}\right)$.

We briefly describe the Robinson-Schensted bijections $G_{r}: H_{n} \rightarrow S D T_{r}(n) \times$ $S D T_{r}(n)$, following [4] and [17]. The algorithm is based on a map $\alpha$ which inserts a domino with label $i$ into a domino tableau given an element $(i, j, \epsilon)$ of $w \in H_{n}$. This insertion map is similar to the usual Robinson-Schensted insertion map and is precisely defined in [4, (1.2.5)]. To construct the left tableau, start with $S_{r}(0)$, the only tableau in $S D T_{r}(0)$. Define $S_{r}(1)=\alpha\left(\left(w_{1}, 1, \epsilon_{1}\right), S_{r}(0)\right)$ and continue inductively by letting

$$
S_{r}(k+1)=\alpha\left(\left(w_{k+1}, k+1, \epsilon_{k+1}\right), S_{r}(k)\right) .
$$

The left domino tableau $S_{r}(n)$ will be standard and of rank $r$. The right tableau is defined to track the shape of the left tableau. Begin by forming a domino tableau $T_{r}(1)$ by adding a domino with label 1 to $S_{r}(0)$ in such a way that $S_{r}(1)$ and $T_{r}(1)$ have the same shape. Continue adding dominos by requiring that at each step $T_{r}(k)$ lie in $S D T_{r}(k)$ and have the same shape as $S_{r}(k)$. Again, the domino tableau $T_{r}(n)$ will be standard and of rank $r$. We define the Robinson-Schensted map $G_{r}(w)=$ $\left(S_{r}(n), T_{r}(n)\right)$ and will write $S_{r}(w)=S_{r}(n)$ and $T_{r}(w)=T_{r}(n)$ for the left and right tableaux.

When $r=0$ or 1 , the $G_{r}$ are precisely Garfinkle's algorithms; for $r>1$ they are natural extensions to larger-rank tableaux. In all cases, $G_{r}$ defines a bijection from $H_{n}$ to pairs of same-shape tableaux in $S D T_{r}(n)$ (see [17]). According to [17, (4.2)], 
$G_{r}\left(w^{-1}\right)=(T, S)$ whenever $G_{r}(w)=(S, T)$. In particular, $w$ is an involution iff $G_{r}(w)=(S, S)$ for some standard domino tableau $S$.

There is a natural description of the relationship between the bijections $G_{r}$ for differing $r$ which we recount at the end of the next section. We also point out that for $r$ sufficiently large, $G_{r}$ recovers the algorithm $H$ mentioned in the introduction (see [16] and [13]).

\subsection{Cycles}

We now review the notion of a cycle in a domino tableau. It appears in a number of references. See for instance [4] and [18] as well as [3] and [19].

For $T \in S D T_{r}(n)$ we will call the square $s_{i j}$ fixed if $i+j$ has the opposite parity as $r$, otherwise, we'll call it variable. If $s_{i j}$ is variable and $i$ is odd, we will say $s_{i j}$ is of type $\mathrm{X}$; if $i$ is even, we will say $s_{i j}$ is of type W. We will write $D(k, T)$ for the domino labeled by the positive integer $k$ in $T$ and $\operatorname{supp} D(k, T)$ will denote its underlying squares. Write label $s_{i j}$ for the label of the square $s_{i j}$ in $T$. We extend this notion slightly by letting label $s_{i j}=0$ if either $i$ or $j$ is less than or equal to zero, and label $s_{i j}=\infty$ if $i$ and $j$ are positive but $s_{i j}$ is not a square in $T$.

Definition 2.1 Suppose that $\operatorname{supp} D(k, T)=\left\{s_{i j}, s_{i+1, j}\right\}$ or $\left\{s_{i, j-1}, s_{i j}\right\}$ and the square $s_{i j}$ is fixed. Define $D^{\prime}(k)$ to be a domino labeled by the integer $k$ with supp $D^{\prime}(k, T)$ equal to $\left\{s_{i j}, s_{i-1, j}\right\}$ if $k<$ label $s_{i-1, j+1}$ and $\left\{s_{i j}, s_{i, j+1}\right\}$ if $k>$ label $s_{i-1, j+1}$. Alternately, suppose that $\operatorname{supp} D(k, T)=\left\{s_{i j}, s_{i-1, j}\right\}$ or $\left\{s_{i, j+1}, s_{i j}\right\}$ and the square $s_{i j}$ is fixed. Define supp $D^{\prime}(k, T)$ to be $\left\{s_{i j}, s_{i, j-1}\right\}$ if $k<$ label $s_{i+1, j-1}$ and $\left\{s_{i j}, s_{i+1, j}\right\}$ if $k>$ label $s_{i+1, j-1}$.

Definition 2.2 The cycle $c=c(k, T)$ through $k$ in a standard domino tableau $T$ is a union of labels of dominos in $T$ defined by the condition that $l \in c$ if either $l=k$, or either supp $D(l, T) \cap \operatorname{supp} D^{\prime}(m, T) \neq \emptyset$ or supp $D^{\prime}(l, T) \cap \operatorname{supp} D(m, T) \neq \emptyset$ for some $D(m, T) \in c$.

We will often refer to the set of dominos with labels in a cycle $c$ as the cycle $c$ itself. For a standard domino tableau $T$ of rank $r$ and a cycle $c$ in $T$, define a domino tableau $M T(T, c)$ by replacing every domino $D(l, T) \in c$ by the corresponding domino $D^{\prime}(l, T)$.

The tableau $M T(T, c)$ is standard, and in general, the shape of $M T(T, c)$ will either equal the shape of $T$, or one square will be removed (or added to the core) and one will be added $[4,(1.5 .27)]$. A cycle $c$ is called closed in the former case and open in the latter. We will write $O C(T)$ for the set of open cycles in $T$. For $c \in O C(T)$, we will write $S_{b}(c)$ for the square that is either removed from the shape of $T$ or added to the core of $T$ by moving through $c$. Similarly, we will write $S_{f}(c)$ for the square that is added to the shape of $T$. Note that $S_{b}(c)$ and $S_{f}(c)$ are always variable squares.

Definition 2.3 A variable square $s_{i j}$ in $\partial(T) \cup \rho(T)$ with the property that neither $s_{i, j+1}$ nor $s_{i+1, j}$ lie in $T$ will be called a hole if it is of type $\mathrm{W}$ and a corner if it is of type $X$. 
We will write $\Delta(T)$ for the cycles through $\delta(T)$. The squares $S_{b}(c)$ and $S_{f}(c)$ are of the same type if $c \in \Delta(T)$. However, for a cycle $c \notin \Delta(T)$, one of $S_{b}(c)$ and $S_{f}(c)$ must be a corner and the other a hole. If the row number of $S_{b}(c)$ is smaller than the row number of $S_{f}(c)$, we will call $c$ a down cycle; otherwise, we will say $c$ is an up cycle.

Let $U$ be a set of cycles in $T$. According to [4, (1.5.29)], the order in which one moves through a set of cycles does not matter, allowing us to unambiguously write $M T(T, U)$ for the tableau obtained by moving-through all of the cycles in the set $U$. Moving through a cycle in a pair of same-shape tableaux is a slightly more delicate operation and requires the following definition (see [5, (2.3.1)]).

Definition 2.4 Consider $(S, T)$ a pair of same-shape domino tableaux, $k$ a label in $S$, and $c$ the cycle in $S$ through $k$. The extended cycle $\tilde{c}$ of $k$ in $S$ relative to $T$ is a union of cycles in $S$ which contains $c$. Further, the union of two cycles $c_{1} \cup c_{2}$ lies in $\tilde{c}$ if either is contained in $\tilde{c}$ and, for some cycle $d$ in $T, S_{b}(d)$ coincides with a square of $c_{1}$ and $S_{f}(d)$ coincides with a square of $M T\left(S, c_{2}\right)$. The symmetric notion of an extended cycle in $T$ relative to $S$ is defined in the natural way.

Let $\tilde{c}$ be an extended cycle in $T$ relative to $S$. According to the definition, it is possible to write $\tilde{c}=c_{1} \cup \ldots \cup c_{m}$ and find cycles $d_{1}, \ldots, d_{m}$ in $S$ such that $S_{b}\left(c_{i}\right)=$ $S_{b}\left(d_{i}\right)$ for all $i, S_{f}\left(d_{m}\right)=S_{f}\left(c_{1}\right)$, and $S_{f}\left(d_{i}\right)=S_{f}\left(c_{i+1}\right)$ for $1 \leq i<m$. The union $\tilde{d}=d_{1} \cup \ldots \cup d_{m}$ is an extended cycle in $S$ relative to $T$ called the extended cycle corresponding to $\tilde{c}$. Symmetrically, $\tilde{c}$ is the extended cycle corresponding to $\tilde{d}$.

We define a moving through operation for a pair of same-shape domino tableaux. If we let $b$ be the ordered pair $(\tilde{c}, \tilde{d})$ of extended cycles in $(S, T)$ that correspond to each other, then we define

$$
M T((S, T), b)=(M T(S, \tilde{c}), M T(T, \tilde{d})) .
$$

As desired, this operation produces another pair of same-shape domino tableaux [5, (2.3.1)].

Finally, we are ready to describe the relationship between the Robinson-Schensted maps $G_{r}$ introduced in the previous section. Recall that the set $\delta(T)$ consists of the squares $s_{i j}$ of $T$ adjacent to the core of $T$, and $\Delta(T)$ is the set of open cycles in $T$ that pass through the squares in $\delta(T)$. Let $\gamma(S)$ be the extended cycles in $S$ relative to $T$ that pass through $\delta(S)$; define $\gamma(T)$ similarly. If we write $\gamma$ for the pair $(\gamma(S), \gamma(T))$, then

$$
\operatorname{MMT}((S, T)) \equiv M T((S, T), \gamma)
$$

is the minimal moving through map that clears all of the squares in $\delta(S)$ and $\delta(T)$ simultaneously.

Theorem 2.5 [15] Consider an element $w \in H_{n}$. The Robinson-Schensted maps $G_{r}$ and $G_{r+1}$ for rank $r$ and $r+1$ domino tableaux are related by

$$
G_{r+1}(w)=\operatorname{MMT}\left(G_{r}(w)\right) .
$$




\subsection{Cycle structure}

Let us define a few objects that will be useful in describing the cycle structure of a domino tableau $T$.

Definition 2.6 Let $s_{i j}$ and $s_{k l}$ lie in the set of corners and holes of $T$. We will say that $s_{m n}$ is between $s_{i j}$ and $s_{k l}$ iff $m$ is between $i$ and $k$ and $n$ is between $j$ and $l$ (where $m$ is between $i$ and $k$ iff $i \leq m \leq k$ or $i \geq m \geq k$ ). We will also say $s_{i j}$ is above $s_{k l}$ if $i<k$.

We will say that a cycle $d \in \Delta(T)$ is adjacent to a cycle $c \in O C^{*}(T)=O C(T) \backslash$ $\Delta(T)$ if there is no cycle $d^{\prime} \in \Delta(T)$ such that $S_{f}\left(d^{\prime}\right)$ is between $S_{f}(d)$ and $S_{f}(c)$.

The set of non-core open cycles $O C^{*}(T)$ has a partial order defined by $c^{\prime} \succeq c^{\prime \prime}$ iff $S_{f}\left(c^{\prime \prime}\right)$ is between $S_{b}\left(c^{\prime}\right)$ and $S_{f}\left(c^{\prime}\right)$. Let $\mu(T)=\left\{c_{1}, c_{2}, \ldots\right\}$ be the set of maximal elements in this poset. For every $c \in \mu(T)$, we will write $c_{\succeq}$ for the set of cycles smaller than or equal to $c$. We also form a rooted tree $\tau(c)$ whose vertices correspond to cycles $c^{\prime} \in c_{\succeq}$, each labeled by 0 if $c^{\prime}$ is an up cycle and 1 if it is a down cycle. Edges in the tree $\tau(c)$ are defined in the natural way from the Hasse diagram of the poset, and for cycles of the same depth we place $c^{\prime}$ to the left of $c^{\prime \prime}$ if $S_{b}\left(c^{\prime}\right)$ is above $S_{b}\left(c^{\prime \prime}\right)$. Finally, $\tau(T)$ will denote the ordered set of trees $\left(\tau\left(c_{1}\right), \tau\left(c_{2}\right), \ldots\right)$ where $S_{b}\left(c_{i}\right)$ is above $S_{b}\left(c_{i+1}\right)$.

Example 2.7 Consider the domino tableau

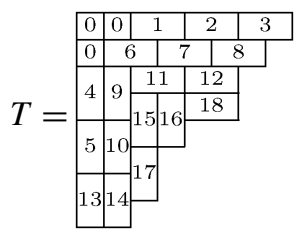

It has three open cycles in the set $O C(T) \backslash \Delta(T): c_{1}=\{9,10,11,12,14\}, c_{2}=\{17\}$, and $c_{3}=\{18\}$. The set $\mu(T)$ contains only the cycle $c_{1}$ and $\tau(T)=\left(\tau\left(c_{1}\right)\right)$ where:

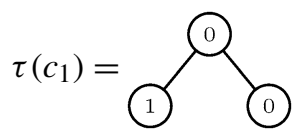

We will use $\tau(T)$ to keep track of the relative positions of the non-core open cycles in $T$. We will also need to keep track of the exact locations of the beginning and final squares of all the open cycles in $T$, for which we use the notion of a cycle structure set.

Definition 2.8 For a standard domino tableau $T$, we define the cycle structure set of $T$ as the set of ordered pairs $c s(T)$ consisting of the beginning and final squares of every cycle in $T$. That is:

$$
c s(T)=\left\{\left(S_{b}(c), S_{f}(c)\right) \mid c \in O C(T)\right\} .
$$


In general, the open cycles of a standard domino tableau can have fairly complicated shapes. However, for any $T$, it is always possible to find another standard domino tableau with hook-shaped open cycles and the same cycle structure set.

Definition 2.9 We will say that an open cycle $c$ in $T$ is hook-shaped iff the set of its underlying squares is entirely contained in the union of one row and one column of $T$.

Proposition 2.10 For every standard domino tableau $T$, there exists another standard domino tableau $\Gamma(T)$ of the same shape such that:

- $\operatorname{cs}(\Gamma(T))=\operatorname{cs}(T)$

- every $c \in O C(\Gamma(T))$ is hook-shaped.

Proof We first show that an appropriate domino tiling $d(T)$ of shape $(T)$ is possible and then show that its dominos can be labeled as a standard tableau $\Gamma(T)$ with the required cycle structure.

We begin by assigning a hook in $\operatorname{shape}(T)$ to every $c \in O C(T)$. Write $S_{b}(c)=s_{i j}$ and $S_{f}(c)=s_{k l}$. For $c$ in $\Delta(T), k-i$ and $l-j$ are both even, implying that the hook with ends $s_{i j}$ and $s_{k, l-1}$ and corner $s_{k j}$ as well as the hook with ends $s_{i j}$ and $s_{k-1, l}$ and corner $s_{i l}$ can both be tiled by dominos. If the domino in the cycle $c$ of $T$ adjacent to the core is vertical, choose the former hook, otherwise, choose the latter. For $c$ in $O C^{*}(T), k-i$ and $l-j$ are both odd, again implying that the hook with ends $s_{i j}$ and $s_{k, l-1}$ and corner $s_{k j}$ as well as the hook with ends $s_{i j}$ and $s_{k-1, l}$ and corner $s_{i l}$ can be tiled by dominos. If $c$ is a down cycle, choose the former hook, otherwise, choose the latter. We will write $h(c)$ for the set of dominos in the hook constructed in this manner starting with a cycle $c \in O C(T)$. It is not hard to see that it is possible to tile the above hook-shapes with dominos for all cycles in $O C(T)$ simultaneously.

Next, we note that the remaining squares of $\operatorname{shape}(T)$ can be tiled with 2-by2 shapes. To be more explicit, we make the following definitions for the hooks in shape $(T)$ constructed above. Two such hooks will be said to have adjacent vertical components if for every pair of squares of the form $\left\{s_{i j}, s_{i l}\right\}$ among the two hooks, there is no other hook containing a square of the form $s_{i m}$ with $m$ between $j$ and $l$. A vertical component containing a square $s_{i j}$ with a property that no other hook contains a square of the form $s_{i l}$ with $l$ greater than $j$ will be said to be adjacent to the boundary of shape $(T)$. We also make the analogous definitions for the horizontal components of the above hooks. Since adjacent corners and holes in $T$ have different types, adjacent vertical components of two hooks must be separated by an even number of columns, and vertical components adjacent to the boundary of shape $(T)$ must be separated from it by an even number of columns. Similarly, adjacent horizontal components of two hooks must be separated by an even number of rows and horizontal components adjacent to the boundary of $\operatorname{shape}(T)$ must be separated from it by an even number of rows. This observation permits us to tile the remaining squares of shape $(T)$ with 2-by-2 shapes.

Using the above observations, define a domino tiling $d(T)$ of shape $(T)$ as the set of $h(c)$ for all $c \in O C(T)$ together with a tiling of the remaining 2-by-2 shapes with pairs of adjacent vertical dominos. 
Next, we show that $d(T)$ can be numbered to create a standard domino tableau. To simplify the description, we first make the following construction.

Definition 2.11 Let $e$ be a domino which intersects $\partial(D)$ of a domino-tiled skew diagram $D$ of shape $\lambda \backslash \mu$. We construct a rooted tree $t(e)$ with vertices corresponding to a certain subset $v(e)$ of the dominos in the tiling of $D$. The domino $e$ will correspond to the root of the tree. The rest of $t(e)$ is constructed recursively.

So suppose that $f$ is a domino of $D$ which corresponds to the vertex $v$. Whenever $f$ is either a horizontal domino containing the corner $s_{i j}$ of a hook $h(c)$ with $c \in$ $O C^{*}(T)$, or a domino in a vertical portion of a hook $h(c)$ for $c \in O C(T)$ with bottom square $s_{i j}$ fixed, we define $\tilde{f}$ as the domino containing $s_{i+1, j-1}$. We say that one domino is adjacent to another if two of their underlying squares share a side. The set of children of $v$ in $t(e)$ consists of all the dominos adjacent to the left side of $f$ together with the domino $\tilde{f}$ whenever it is defined. If there is only one child of $f$, place it as a right child of $f$ in $t(e)$. Otherwise, order the children left to right with the top-most domino as the left child. This process then can be continued until the leaves of $t(e)$ correspond to dominos adjacent to the boundary of $\mu$.

We are now ready to describe how to number the dominos of $d(T)$. Consider the domino $e$ at the top edge of $\partial(T)$. The vertices of $t(e)$ can be numbered with the integers $\{1,2, \ldots,|t(e)|\}$ according to a postfix order traversal, and the labels of $t(e)$ can be transferred to $d(T)$. The entire process can then be repeated for the skew diagram $d(T) \backslash v(e)$ with labels starting at $|t(e)|+1$. Iterating this procedure until $d(T)$ is exhausted yields a numbered domino tiling of $d(T)$. It is not difficult to verify that it is in fact a standard domino tableau which we call $\Gamma(T)$.

Finally, we check that $\Gamma(T)$ has the desired cycles. First, we show that all dominos of $\Gamma(T)$ not contained in a hook $h(c)$ for some $c \in O C(T)$ lie in a closed cycle of size 2 in $\Gamma(T)$. Consider the dominos of $\Omega=\Gamma(T) \backslash\{h(c) \mid c \in O C(T)\}$. Choose a domino $e_{1} \in \Omega$ whose top and left edges are not adjacent to any other members of $\Omega$. Its top square $s_{i j}$ is necessarily fixed. Further, the label of $s_{i-1, j+1}$ is necessarily smaller than the label of $s_{i j}$ since it either lies outside of shape $(T)$, or the domino containing it corresponds to either a vertex on a prior branch of its tree, or even a tree that has been labeled previously. Hence $M T\left(e_{1}, \Gamma(T)\right)$ consists of the squares $\left\{s_{i j}, s_{i, j+1}\right\}$. The domino $e_{2}=\left\{s_{i, j+1}, s_{i+1, j+1}\right\}$ of $\Gamma(T)$ has fixed square $s_{i+1, j+1}$. Its label is necessarily smaller then the label of $s_{i+2, j}$, since the latter square either lies outside the Young diagram underlying shape $(T)$, or the domino containing it corresponds to either a vertex on a later branch of its tree, or even a tree labeled later. Hence $M T\left(e_{2}, \Gamma(T)\right)$ consists of the squares $\left\{s_{i+1, j}, s_{i+1, j+1}\right\}$ and $\left\{e_{1}, e_{2}\right\}$ is a closed cycle in $\Gamma(T)$. This argument can be repeated again with $\Omega$ replaced with $\Omega \backslash\left\{e_{1}, e_{2}\right\}$ until $\Omega$ is exhausted.

Now, consider a hook $h(c)$ corresponding to $c \in O C(T)$. When labeled, it constitutes an open cycle of $\Gamma(T)$; the fact that if $e$ is a domino in $h(c)$, then the squares of $M T(e, T)$ again lie in $h(c)$ follows directly from our method of numbering $d(T)$. Furthermore, if $c \in O C(T)$, then $S_{b}(h(c))=s_{i j}=S_{b}(c)$ and $S_{f}(h(c))=$ $s_{k l}=S_{f}(c)$. Since the $h(c)$ are the only open cycles of $\Gamma(T)$, we can conclude that $\operatorname{cs}(\Gamma(T))=\operatorname{cs}(T)$. 
Example 2.12 We provide an example of the method of labeling the dominos in a domino diagram to form a standard domino tableau used in the above construction. Consider the domino diagram $E$ of shape $\{5,5,4,3\} \backslash\{2,1\}$. The domino $e$ is at the top of its boundary:

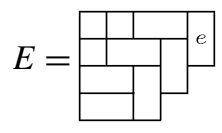

The dominos in $E$ can be represented in the rooted tree $t(e)$ which can be numbered according to a postfix traversal order as below.


Finally, this numbering of the vertices of the above tree yields the standard domino tableau $T$ :

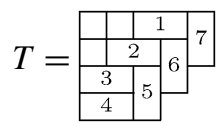

Note that in the proof of the previous proposition, the only data required from $T$ to construct $\Gamma(T)$ was its shape and the cycle structure set $\operatorname{cs}(T)$. Thus given a shape $\lambda$ of a domino tableau and a set $c s$ of pairs of beginning and final squares, we will write $\Gamma(\lambda, c s)$ for the standard domino tableau constructed via the above process.

\section{Equivalence relations on $W$}

We define two equivalence relations on domino tableaux in $S D T_{r}(n)$. Both can be used to define equivalence classes in $W$ of type $B_{n}$ by using the generalized Robinson-Schensted algorithms $G_{r}$. The main result is that the equivalence classes thus defined are in fact the same.

Definition 3.1 Consider $T, T^{\prime} \in S D T_{r}(n)$. We say $T \sim_{r} T^{\prime}$ iff there is a subset of non-core open cycles $U \subset O C^{*}\left(T^{\prime}\right)$ such that $T=M T\left(T^{\prime}, U\right)$.

It is not difficult to see that $\sim_{r}$ defines an equivalence relation on $S D T_{r}(n)$. We will use it to define an equivalence relation on $W$. First, note that elements of an $\sim_{r}$-equivalence class of $T$ correspond to $\{0,1\}$-labelings of the vertices of the trees underlying $\tau(T)$. Such a labeling determines a unique standard domino tableau $T^{\prime}$ in the equivalence class of $T$.

Definition 3.2 Consider $w, y \in W$ and let $w \sim_{r} y$ iff $T_{r}(w) \sim_{r} T_{r}(y)$.

Hence the equivalence relation $\sim_{r}$ on $W$ is completely determined by an equivalence relation on right tableaux. 
Definition 3.3 Consider $T, T^{\prime} \in S D T_{r}(n)$. We will say $T \varkappa_{r}^{\prime} T^{\prime}$ iff there exist $S, S^{\prime} \in S D T_{r}(n)$ such that $\operatorname{MMT}(S, T)$ and $\operatorname{MMT}\left(S^{\prime}, T^{\prime}\right)$ have the same right tableau. We will let $\leftrightarrow r$ be its transitive closure.

Definition 3.4 Consider $w, y \in W$ and define a relation $w \varkappa_{r}^{\prime} y$ iff $T_{r}(w)=T_{r}(y)$ or $T_{r+1}(w)=T_{r+1}(y)$. Let $\varkappa_{r}$ be its transitive closure.

Both of the relations denoted by $\leadsto r$ are in fact equivalence relations. Again, the equivalence relation $\leadsto r$ on $W$ can be completely expressed as an equivalence relation on right tableaux, mirroring Definition 3.2.

Proposition 3.5 Consider $w, y \in W$, then $w \rightsquigarrow r$ iff $T_{r}(w) \leftrightarrow \rightsquigarrow_{r} T_{r}(y)$.

Proof Let's simplify the notation and write $\sim$ for $\sim_{r}$ and $\leadsto$ for $\rightsquigarrow_{r}$. Note that $w w^{\prime} y$ means that either $T_{r}(w)=T_{r}(y)$, in which case $T_{r}(w) \leftrightarrow \iota^{\prime} T_{r}(y)$, or that $T_{r+1}(w)=T_{r+1}(y)$. In the latter case, the description of the map $M M T$ implies that $M M T\left(G_{r}(w)\right)$ and $M M T\left(G_{r}(y)\right)$ have the same right tableaux and $T_{r}(w) \longleftrightarrow{ }^{\prime}$ $T_{r}(y)$. Hence the relation $\leadsto$ on $W$ implies the relation $\leadsto$ on tableaux.

Conversely, suppose that $T_{r}(w) \mathrm{s}^{\prime} T_{r}(y)$. Consider tableaux $S$ and $S^{\prime}$ such that $\operatorname{MMT}\left(S, T_{r}(w)\right)$ and $M M T\left(S^{\prime}, T_{r}(y)\right)$ have the same right tableaux. If we let $w^{\prime}=G_{r}^{-1}\left(S, T_{r}(w)\right)$ and $y^{\prime}=G_{r}^{-1}\left(S^{\prime}, T_{r}(y)\right)$, then $w^{\prime} x^{\prime} y^{\prime}$ since $G_{r+1}\left(w^{\prime}\right)=$ $\operatorname{MMT}\left(S, T_{r}(w)\right)$ and $G_{r+1}\left(y^{\prime}\right)=\operatorname{MMT}\left(S^{\prime}, T_{r}(y)\right)$. Finally, $T_{r}(w)=T_{r}\left(w^{\prime}\right)$ and

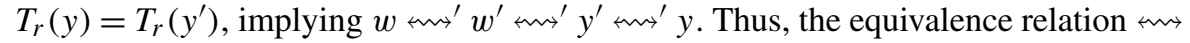
on tableaux implies the relation $\leadsto$ on $W$.

Our main result states that $\sim_{r}$ and $\rightsquigarrow_{r}$ in fact define the same equivalence classes in $W$.

Theorem 3.6 Consider $w, y \in W$, then $w \sim_{r} y$ iff $w \rightsquigarrow r y$.

Proof Again, let's write $\sim$ for $\sim_{r}$ and $\leadsto$ for $\rightsquigarrow_{r}$. The fact that $w \rightsquigarrow y$ implies $w \sim y$ is a consequence of the relationship between the Robinson-Schensted maps $G_{r}$ and $G_{r+1}$ described in Theorem 2.5.

If $w$ sn' $y$, then either $T_{r}(w)=T_{r}(y)$ or $T_{r+1}(w)=T_{r+1}(y)$. In the former case, we automatically have $w \sim y$. In the latter case, $T_{r+1}(w)=T_{r+1}(y)$ and Theorem 2.5 implies that $\operatorname{MMT}\left(G_{r}(w)\right)$ and $M M T\left(G_{r}(y)\right)$ have the same right tableaux. According to the definition of the $M M T$ map,

$$
T_{r+1}(w)=M T\left(T_{r}(w), \Delta\left(T_{r}(w)\right) \cup X\right)
$$

for a subset of non-core open cycles $X \subset O C^{*}\left(T_{r}(w)\right)$ and

$$
T_{r+1}(y)=M T\left(T_{r}(y), \Delta\left(T_{r}(y)\right) \cup Y\right)
$$

for a subset of non-core open cycles $Y \subset O C^{*}\left(T_{r}(y)\right)$. Since the moving-through operation can be performed on disjoint sets of cycles independently, $M T\left(T_{r}(w), X\right)$ 
must equal $M T\left(T_{r}(y), Y\right)$. In particular, this forces $T_{r}(w)=M T\left(T_{r}(y), U\right)$ for some subset $U \subset O C^{*}\left(T_{r}(y)\right)$, implying $T_{r}(w) \sim T_{r}(y)$ and $w \sim y$.

Our proof that $w \sim y$ implies $w \leadsto y$ requires the following lemma, which we verify in the following section.

Lemma 3.7 Suppose that $T^{\prime \prime}=M T\left(T^{\prime}, U\right)$ for some subset $U \subset O C^{*}\left(T^{\prime}\right)$, and that the labels of $\tau\left(T^{\prime}\right)$ and $\tau\left(T^{\prime \prime}\right)$ disagree only on one tree. Then $T^{\prime} \leadsto T^{\prime \prime}$.

Let $T=T_{r}(w)$ and $T^{\prime}=T_{r}(y)$. By definition, $w \sim y$ implies $T^{\prime}=M T(T, U)$ for some subset $U$ of non-core open cycles of $T$. Let's suppose that the positions of the cycles of these tableaux are given by the trees $\tau(T)=\left(\tau_{1}, \tau_{2}, \ldots, \tau_{m}\right)$ and $\tau\left(T^{\prime}\right)=\left(\tau_{1}^{\prime}, \tau_{2}^{\prime}, \ldots, \tau_{m}^{\prime}\right)$. Using the note following Definition 3.1, construct domino tableaux $T_{i}$ with $\tau\left(T_{i}\right)=\left(\tau_{1}, \tau_{2}, \ldots, \tau_{i}, \tau_{i+1}^{\prime}, \ldots \tau_{m}^{\prime}\right)$. According to Lemma 3.7, we have constructed a sequence of tableaux $\left\{T_{i}\right\}$ satisfying

$$
T^{\prime}=T_{0} \leadsto T_{1} \leadsto T_{2} \leadsto \ldots \leftrightarrow T_{m}=T .
$$

The characterization of $\leadsto$ given in Proposition 3.5 implies $w \rightsquigarrow y$, as desired.

\section{Main lemma}

This section details the proof of Lemma 3.7. We retain the notation used therein and begin with a definition and a result on $\{0,1\}$-labeled trees.

Definition 4.1 Suppose $\tau^{\prime}$ and $\tau^{\prime \prime}$ are $\{0,1\}$-labeled trees as in Section 2.4 which share the same underlying unlabeled embedded rooted tree $\varsigma$. For $\epsilon \in\{0,1\}$, we say $\tau^{\prime} \leftrightarrow{ }_{\epsilon}^{\prime} \tau^{\prime \prime}$ if there is a path $\kappa$ in $\zeta$ satisfying

(1) $\kappa$ contains the root of $\zeta$

(2) labels of $\tau^{\prime}$ and $\tau^{\prime \prime}$ agree on the vertices of $\varsigma \backslash \kappa$,

(3) except for the vertex of greatest depth in $\kappa$, the labels of vertices in $\kappa$ in $\tau^{\prime}$ and $\tau^{\prime \prime}$ agree, alternate, and begin with $\epsilon$ at the root.

We will write $\rightsquigarrow_{\epsilon}$ for the transitive closure of $\varkappa_{\epsilon}^{\prime}$.

We note that according to the above, a path $\kappa$ can contain just the root of $\varsigma$. The next result shows that for each $\varsigma$, there is only one equivalence class of $\{0,1\}$-labeled trees. While this means that the relation $\rightsquigarrow_{\epsilon}$ is trivial, we've introduced it as we will need to know the sequence of ${ }^{\prime}{ }_{\epsilon}^{\prime}$ that accomplishes this.

Proposition 4.2 Two $\{0,1\}$-labeled trees which share the same underlying rooted tree are $\aleph_{\epsilon}$-equivalent.

Proof Consider two $\{0,1\}$-labeled trees $\tau^{\prime}$ and $\tau^{\prime \prime}$ which share the same underlying unlabeled rooted tree $\zeta$. Let $\tau_{a}$ be a labeling of the vertices of $\varsigma$ with vertices of even depth labeled by $\epsilon$ and vertices of odd depth labeled by $1-\epsilon$. Starting with the set of vertices of maximal depth $m$ in $\varsigma$ and using the definition of $m_{\epsilon}^{\prime}$, we can find a 
sequence of trees which agree with $\tau_{a}$ on vertices of depth less than $m$ and are ${ }^{\prime} \epsilon^{-}$ equivalent to the tree whose labels agree with $\tau_{a}$ on vertices of depth less than $m$ and vertices of $\tau^{\prime}$ on maximal depth vertices. This procedure can be repeated successively for smaller depths, creating a sequence

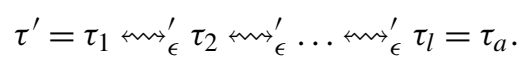

The above procedure can be repeated with $\tau^{\prime}$ replaced with $\tau^{\prime \prime}$, finally creating the desired sequence of labeled embedded trees

$$
\tau^{\prime}=\tau_{1} \leadsto{ }_{\epsilon}^{\prime} \tau_{2} \rightsquigarrow_{\epsilon}^{\prime} \ldots \varkappa_{\epsilon}^{\prime} \tau_{m}=\tau^{\prime \prime} .
$$

Example 4.3 We exhibit the above procedure for the trees $\tau^{\prime}$ and $\tau^{\prime \prime}$ and $\epsilon=1$. Here, $\tau_{a}$ is the third tree in the sequence.
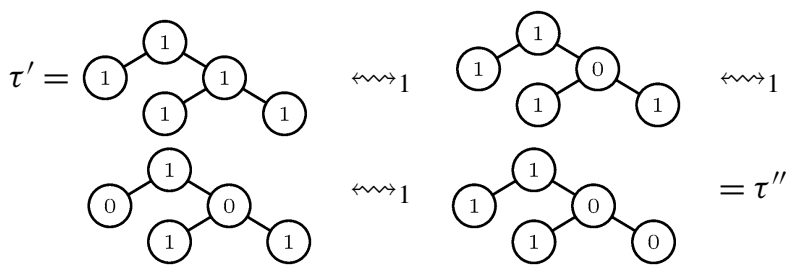

We are ready to start the proof of the main lemma. Suppose that $T^{\prime \prime}=M T\left(T^{\prime}, U\right)$ for some subset $U \subset O C^{*}\left(T^{\prime}\right)$, and that the labels of $\tau\left(T^{\prime}\right)$ and $\tau\left(T^{\prime \prime}\right)$ disagree only on the $(k+1)$-st tree:

$$
\begin{aligned}
\tau\left(T^{\prime}\right) & =\left(\tau^{1}, \tau^{2}, \ldots, \tau^{k}, \tau^{\prime}, \tau^{k+2}, \ldots\right) \\
\tau\left(T^{\prime \prime}\right) & =\left(\tau^{1}, \tau^{2}, \ldots, \tau^{k}, \tau^{\prime \prime}, \tau^{k+2}, \ldots\right)
\end{aligned}
$$

Write $c$ for the cycle of $T^{\prime}$ and $T^{\prime \prime}$ that corresponds to the root of both the trees $\tau^{\prime}$ and $\tau^{\prime \prime}$, and choose a cycle $d \in \Delta\left(T^{\prime}\right)=\Delta\left(T^{\prime \prime}\right)$ adjacent to $c$ (see Section 2.4). If $S_{f}(d)$ is above $S_{f}(c)$, then take $\epsilon=1$. Otherwise, let $\epsilon=0$. The previous proposition gives $\tau^{\prime} \rightsquigarrow \epsilon \tau^{\prime \prime}$ via the sequence

$$
\tau^{\prime}=\tau_{1} \leadsto{ }_{\epsilon}^{\prime} \tau_{2}{ }_{\epsilon}^{\prime} \ldots{ }_{\epsilon}^{\prime} \tau_{m}=\tau^{\prime \prime} .
$$

Using the note following Definition 3.1, we can construct a sequence of tableaux $\left\{T_{i}\right\}_{i=1}^{m}$ from this sequence of labeled embedded trees, each satisfying

$$
\tau\left(T_{i}\right)=\left(\tau^{1}, \tau^{2}, \ldots, \tau^{k}, \tau_{i}, \tau^{k+2}, \ldots\right) .
$$

Claim 4.1 With $\epsilon$ as above, $T_{i} \leftrightarrow{ }^{\prime} T_{i+1}$.

Proof Since $\tau_{i} \leadsto{ }_{\epsilon}^{\prime} \tau_{i+1}$, we must have $T_{i+1}=M T\left(T_{i}, \tilde{c}\right)$ for some cycle $\tilde{c} \preceq c$. Let $c=c_{1}, c_{2}, \ldots c_{l}=\tilde{c}$ be the maximal chain between them in the poset of open cycles. Except perhaps for $\tilde{c}$, it alternates between up and down cycles in both $T_{i}$ and $T_{i+1}$ because of the way $\rightarrow^{\prime}$ is defined. Furthermore, unless $l=1, c_{1}$ must be an up cycle 
if $\epsilon=1$ and a down cycle if $\epsilon=0$. Let $\mathcal{B}$ be the cycle structure set of $T_{i}$ with pairs of squares that correspond to cycles in $\tau_{i}$ deleted. Write $c_{0}=d$ and for $k \geq 1$, let

$$
\begin{aligned}
\mathcal{C}_{k}=\left(S_{b}\left(c_{0}\right), S_{f}\left(c_{1}\right)\right) & \cup \bigcup_{1 \leq j \leq k / 2}\left(S_{b}\left(c_{2 j}\right), S_{f}\left(c_{2 j-2}\right)\right) \\
& \cup \bigcup_{1 \leq j<k / 2}\left(S_{b}\left(c_{2 j-1}\right), S_{f}\left(c_{2 j+1}\right)\right)
\end{aligned}
$$

and

$$
\mathcal{D}_{k}= \begin{cases}\mathcal{B} \cup \mathcal{C}_{k} \cup\left(S_{b}\left(c_{k}\right), S_{f}\left(c_{k-1}\right)\right) & \text { if } k \text { is odd } \\ \mathcal{B} \cup \mathcal{C}_{k} \cup\left(S_{b}\left(c_{k-1}\right), S_{f}\left(c_{k}\right)\right) & \text { if } k \text { is even. }\end{cases}
$$

When $k=0$, let $\mathcal{D}_{0}=\mathcal{B}$. There are two possibilities for the position of $c_{l}$ in $T_{i}$. First assume that $c_{1}, c_{2}, \ldots, c_{l}$ alternates between up and down cycles. Following Proposition 2.10 and especially the comment after it, we can find standard domino tableaux $S_{i}=\Gamma\left(\operatorname{shape}\left(T_{i}\right), \mathcal{D}_{l}\right)$ and $S_{i}^{\prime}=\Gamma\left(\operatorname{shape}\left(T_{i+1}\right), \mathcal{D}_{l-1}\right)$ satisfying $\operatorname{cs}\left(S_{i}\right)=$ $\mathcal{D}_{l}$ and $\operatorname{cs}\left(S_{i}^{\prime}\right)=\mathcal{D}_{l-1}$. By definition of extended cycles and our choice of $\epsilon$,

$$
\begin{aligned}
e c\left(d, T_{i}, S_{i}\right) & =d \cup c_{1} \cup c_{2} \cup \ldots c_{l}, \text { and } \\
e c\left(d, T_{i+1}, S_{i}^{\prime}\right) & =d \cup c_{1} \cup c_{2} \cup \ldots c_{l-1} .
\end{aligned}
$$

Hence $\operatorname{MMT}\left(S_{i}, T_{i}\right)$ will have right tableau $M T\left(T_{i}, \Delta\left(T_{i}\right) \cup c_{1} \cup c_{2} \cup \ldots c_{l}\right)$ while $\operatorname{MMT}\left(S_{i}^{\prime}, T_{i+1}\right)$ will have right tableau $M T\left(T_{i+1}, \Delta\left(T_{i+1}\right) \cup c_{1} \cup c_{2} \cup \ldots c_{l-1}\right)$. Since $\Delta\left(T_{i+1}\right)=\Delta\left(T_{i}\right)$ and $T_{i+1}=M T\left(T_{i}, c_{l}\right)$, Definition 3.3 implies that $T_{i} \leadsto{ }^{\prime}$ $T_{i+1}$, as desired.

The second possibility for the position of $c_{l}$ in $T_{i}$ is that both $c_{l-1}$ and $c_{l}$ are either up or down cycles. The proof follows as above, but with $S_{i}$ and $S_{i}^{\prime}$ defined to satisfy $\operatorname{cs}\left(S_{i}\right)=\mathcal{D}_{l-1}$ and $\operatorname{cs}\left(S_{i}^{\prime}\right)=\mathcal{D}_{l}$.

Armed with the above claim, we have found a sequence of tableaux $\left\{T_{i}\right\}_{i=1}^{m}$ such that $T_{1}=T^{\prime}, T_{m}=T^{\prime \prime}$ and $T_{i} \leadsto{ }^{\prime} T_{i+1}$ for all $i$, verifying the main lemma.

Example 4.4 We conclude with an example which will hopefully clarify the above procedure. Let
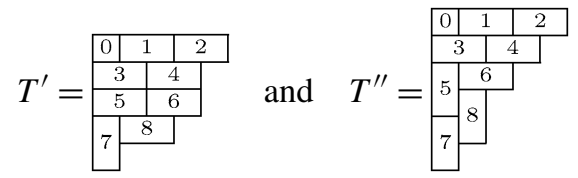

so that $T^{\prime \prime}=M T\left(T^{\prime}, U\right)$, where $U$ consists of all non-core open cycles of $T^{\prime}$. We would like to show that $T^{\prime} \leadsto T^{\prime \prime}$, and so we need to construct a sequence of $m s^{\prime}-$ equivalent tableaux. Both $\tau\left(T^{\prime}\right)$ and $\tau\left(T^{\prime \prime}\right)$ contain only one tree, so we are in the setting of the Lemma 3.7. We first find a sequence of labeled trees which exhibit 
$\tau\left(T^{\prime}\right) \rightsquigarrow 1 \tau\left(T^{\prime \prime}\right)$. The proof of Proposition 4.2 yields the sequence

$$
\tau_{1}=\text { (1) } m 1 \quad \tau_{2}=\underbrace{(1)}_{0} \tau_{3}=\text { (0) }
$$

with $\tau_{1}=\tau\left(T^{\prime}\right)$ and $\tau_{3}=\tau\left(T^{\prime \prime}\right)$. Using the note after Definition 3.1, these trees correspond to the sequence $T_{1}=T^{\prime}, T_{2}$, and $T_{3}=T^{\prime \prime}$ of tableaux with

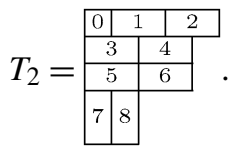

To show that $T_{1}$ «m $T_{2} T_{2}{ }^{\prime} T_{3}$, we need to find tableaux $S_{1}, S_{1}^{\prime}, S_{2}$, and $S_{2}^{\prime}$ which satisfy the equalities $\operatorname{MMT}\left(S_{1}, T_{1}\right)=\operatorname{MMT}\left(S_{1}^{\prime}, T_{2}\right)$ and $\operatorname{MMT}\left(S_{2}, T_{2}\right)=$ $\operatorname{MMT}\left(S_{2}^{\prime}, T_{3}\right)$. This is accomplished in our proof of Claim 4.1 by using Proposition 2.10 to construct tableaux with the required cycle structure:
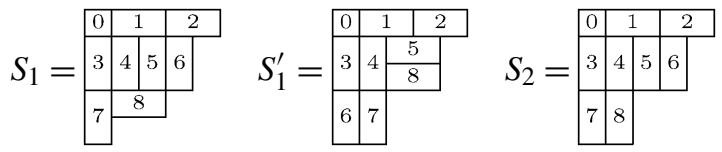

and $S_{2}^{\prime}=T^{\prime \prime}$. We check that

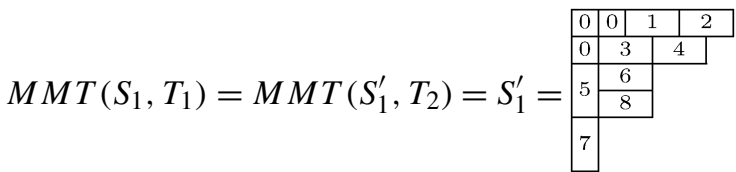

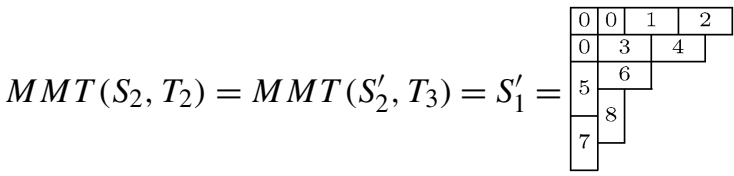

\section{References}

1. Bonnafé, C., Iancu, L.: Left cells in type $B_{n}$ with unequal parameters. Represent. Theory 7, 587-609 (2003)

2. Bonnafé, C., Geck, M., Iancu, L., Lam, T.: On domino insertion and Kazhdan-Lusztig cells in type $B_{n}$. arXiv:math.RT/0609279

3. Carré, C., Leclerc, B.: Splitting the square of a Schur function into its symmetric and anti-symmetric parts. J. Algebr. Comb. 4, 201-231 (1995)

4. Garfinkle, D.: On the classification of primitive ideals for complex classical Lie algebras (I). Compos. Math. 75(2), 135-169 (1990)

5. Garfinkle, D.: On the classification of primitive ideals for complex classical Lie algebras (II). Compos. Math. 81(3), 307-336 (1992)

6. Garfinkle, D.: On the classification of primitive ideals for complex classical Lie algebras (III). Compos. Math. 88(2), 187-234 (1993) 
7. Gordon, I.G., Martino, M.: Calogero-Moser space, reduced rational Cherednik algebras and two-sided cells. arXiv:math.RT/0703153

8. Lusztig, G.: Left cells in Weyl groups. In: Lie Group Representations. Lecture Notes in Mathematics, vol. 1024, pp. 99-111 (1983)

9. Lusztig, G.: Hecke Algebras With Unequal Parameters. CRM Monograph Series, vol. 18. American Mathematical Society, Providence (2003)

10. McGovern, W.M.: Left cells and domino tableaux in classical Weyl groups. Compos. Math. 101(1), 77-98 (1996)

11. McGovern, W.M.: On the Spaltenstein-Steinberg map for classical Lie algebras. Commun. Algebra 27(6), 2979-2993 (1999)

12. McGovern, W.M.: A triangularity result for associated varieties of highest weight modules. Commun. Algebra 28(4), 1835-1843 (2000)

13. Okada, S.: Wreath products by the symmetric groups and product posets of Young's lattices. J. Comb. Theory Ser. A 55(1), 14-32

14. Pietraho, T.: Components of the Springer fiber and domino tableaux. J. Algebra 272(2), 711-729 (2004)

15. Pietraho, T.: A relation for domino Robinson-Schensted algorithms. math.CO/0603654

16. Stanton, D.W., White, D.E.: A Schensted algorithm for rim hook tableaux. J. Comb. Theory Ser. A 40(2), 211-247 (1985)

17. van Leeuwen, M.A.A.: The Robinson-Schensted and Schutzenberger algorithms, an elementary approach. Electron. J. Comb. 3(2), R15 (1996)

18. van Leeuwen, M.A.A.: Edge sequences, ribbon tableaux, and an action of affine permutations. Eur. J. Comb. 20, 397-426 (1999)

19. van Leeuwen, M.A.A.: Some bijective correspondences involving domino tableaux. Electron. J. Comb. 7(1), R20 (2000) 